\title{
Expression of connective tissue growth factor as a prognostic indicator and its possible involvement in the aggressive properties of epithelial ovarian carcinoma
}

\author{
AKIKO SHIMBO $^{1}$, HIROAKI KAJIYAMA ${ }^{1}$, SATOSHI TAMAUCHI ${ }^{1}$, NOBUHISA YOSHIKAWA ${ }^{1}$, YOSHIKI IKEDA ${ }^{1}$, \\ KIMIHIRO NISHINO ${ }^{1}$, SHIRO SUZUKI ${ }^{1}$, KAORU NIIMI $^{1}$, JUN SAKATA ${ }^{2}$ and FUMITAKA KIKKAWA ${ }^{1}$ \\ ${ }^{1}$ Department of Obstetrics and Gynecology, Graduate School of Medicine, Nagoya University, \\ Nagoya, Aichi 466-8550; ${ }^{2}$ Department of Gynecology, Graduate School of Medicine, \\ Aichi Cancer Center Hospital, Nagoya, Aichi 464-8681, Japan
}

Received February 26, 2019; Accepted September 3, 2019

DOI: 10.3892/or.2019.7352

\begin{abstract}
Recently, connective tissue growth factor (CTGF) was demonstrated to be associated with aggressive characteristics, including proliferation, invasion and metastasis, in a number of malignancies. Here, we investigated the expression and function of CTGF in epithelial ovarian carcinoma (EOC) to clarify its molecular mechanism and clinical significance. Paraffin sections from clinical samples of EOC $(\mathrm{N}=104)$ were immunostained with the CTGF antibody, and then the staining positivity was semiquantitatively examined. Moreover, we explored the role of CTGF expression in the migration-promoting effect on and chemoresistance of EOC cells. The results revealed that of the 104 EOC patients, the low and high CTGF staining expression rates were 65 (62.5\%) and $39(37.5 \%)$, respectively. Patients belonging to the higher-level CTGF group showed poorer progression-free (PFS) and overall survival (OS) rates than those in the lower-level group [PFS (log-rank: $\mathrm{P}=0.0076$ ) and OS (log-rank: $\mathrm{P}=0.0078$ ), respectively]. Multivariable analysis showed that CTGF expression was a significant predictor of poorer PFS and OS [PFS: HR (high vs. low): 1.837, 95\% CI: 1.023-3.289 $(\mathrm{P}=0.0418)$; OS: HR: 2.141, 95\% CI: 1.077-4.296 ( $\mathrm{P}=0.0300)]$. In in vitro studies, in acquired paclitaxel (PTX)-resistant EOC cells, the silencing of CTGF expression led to the
\end{abstract}

Correspondence to: Dr Hiroaki Kajiyama, Department of Obstetrics and Gynecology, Graduate School of Medicine, Nagoya University, Tsuruma-cho 65, Showa-ku, Nagoya, Aichi 466-8550, Japan

E-mail: kajiyama@med.nagoya-u.ac.jp

Abbreviations: EOC, epithelial ovarian cancer; HGSOC, high-grade serous ovarian cancer; CTGF, connective tissue growth factor; PFS, progression-free survival; OS, overall survival; FIGO, International Federation of Gynecology and Obstetrics

Key words: epithelial ovarian carcinoma, CTGF, immunohistochemical staining, chemoresistance, recurrence restoration of PTX sensitivity. Furthermore, we confirmed that the TGF- $\beta$-dependent migration-promoting effect on these CTGF-depleted cells was completely inhibited. In conclusion, the results of the present study suggest the possible involvement of CTGF in the migration-promoting effect and chemoresistance of EOC, suggesting that it may be a target for overcoming the malignant properties of EOC.

\section{Introduction}

Based on recent global cancer statistics, 295,414 patients were newly diagnosed with epithelial ovarian carcinoma (EOC) and 184,799 patients died of this tumor worldwide in 2018 (1). Thus, EOC is a leading cause of mortality in women among all gynecologic cancers (2). Since EOC commonly remains asymptomatic, in clinical practice it is known as a 'silent killer', and the majority of patients present with widespread peritoneal metastases at initial diagnosis. The oncologic outcome of EOC patients is likely to be associated with the extent of peritoneal dissemination (3-5). Although complete clinical remission is achieved in about $80 \%$ of patients who undergo aggressive surgery and systematic chemotherapy, most of these clinical responders show recurrence (6). Consequently, the oncologic outcome of recurrent patients remains extremely poor $(7,8)$.

Connective tissue growth factor (CTGF/CCN2) belongs to a member of the CCN family (9). CTGF has been reported to be involved in various aggressive tumor properties, such as cell growth, migration, angiogenesis and metastasis (10-13). Furthermore, according to prior studies, CTGF expression is closely related to the acquisition of chemoresistance to antineoplastic agents, including paclitaxel in breast cancer (14), 5-FU in colorectal cancer (15) and cisplatin in osteosarcoma (16). Regarding EOC, Wang et al revealed that the expression level of CTGF is negatively correlated with the expression of miR-143 in tissue samples, and that miR-143 exerts tumor-suppressing functions, including, migration, invasion and cell proliferation by targeting CTGF in vitro (17). However, to the best of our knowledge, studies concerning the expression and biological behavior of CTGF in relapsed EOC are limited. 
We hypothesized that CTGF plays a central role in both the chemoresistance and metastatic ability of EOC, and that CTGF positivity may be a valuable predictor of a poor clinical outcome in EOC patients. Here, we investigated the prognostic impact of CTGF expression, and analyzed the functions of CTGF in EOC cell progression.

\section{Materials and methods}

Cell culture. The EOC cell lines, ES-2, SKOV3, A2780, and OVCAR3, were maintained in RPMI-1640 medium with $10 \%$ FBS and penicillin/streptomycin. These cell lines were obtained from the American Type Culture Collection (ATCC; Manassas, VA, USA) in 2012-2013. NOS2 and NOS3 cells, derived from serous EOC, were established in our institute $(18,19)$. These cell lines were maintained in RPMI-1640 (Sigma-Aldrich; Merck KGaA) supplemented with $10 \%$ fetal bovine serum (FBS) and penicillin-streptomycin at $37^{\circ} \mathrm{C}$ in a humidified atmosphere of $5 \% \mathrm{CO}_{2}$. The NOS2TR and NOS3TR cells, established from parental NOS2 and NOS3 cells, acquired chronic resistance to paclitaxel (PTX) as previously described (20-22).

Inhibition of CTGF by small interfering RNA (siRNA). To generate CTGF-silenced cells, EOC cells were transfected with either a pool of small interfering RNA (siRNA) oligonucleotide-specific to human CTGF (final concentration, $30 \mathrm{pmol} / \mathrm{l}$; assay ID s3709, cat. no. 4427038; Thermo Fisher Scientific, Inc.) or control siRNA (Sigma-Aldrich; Merck KGaA) using Invitrogen $^{\mathrm{TM}}$ Lipofectamine $^{\mathrm{TM}}$ RNAiMAX Transfection Regent (Thermo Fisher Scientific, Inc.). The sequences for CTGF siRNA were as follows: Sense, 5'-CCUAUCAAGUUU GAGCUUUTT-3' and antisense, 5'-AAAGCUCAAACUUGA UAGGCT-3'. After overnight incubation at $37^{\circ} \mathrm{C}$, the culture medium was replaced with fresh complete medium containing $10 \%$ FBS. Cells were harvested after $72 \mathrm{~h}$ and solubilized for western blot analysis of CTGF silencing.

PTX chemosensitivity assay. The PTX chemosensitivity assay was performed as described previously (23). Briefly, cells were seeded in triplicate in 96-well plates at a density of 5,000 cells in a volume of $200 \mu \mathrm{l}$ of culture media containing $10 \% \mathrm{FBS}$. After incubation for $24 \mathrm{~h}$ at $37^{\circ} \mathrm{C}$, the medium was replaced with fresh medium with or without various concentrations of PTX (Bristol Myers Squib, Tokyo, Japan). After an additional $72 \mathrm{~h}$, cell viability was assayed using the Cell Titer 96 Aqueous One Solution Cell Proliferation Assay kit (Promega Corp., Tokyo, Japan).

In vitro migration assay. Cell migration was assayed in 24-well Transwell cell culture chambers (Costar, Corning Inc., Corning, NY, USA). Cells were suspended in the upper chamber at a final concentration of $1.0 \times 10^{6} / \mathrm{ml}$ in $200 \mu 1$ of RPMI-1640 medium. In addition, we examined the effect of siRNA transfection on the migration of parental and PTX-resistant EOC cells. Cells transfected with siRNAs were seeded in the upper chamber and allowed to migrate to the fibronectin-coated lower surface for $20 \mathrm{~h}$. The number of cells that had migrated to the lower surface was counted to evaluate the migration ability. Cells were seeded in $6-\mathrm{cm}$ dishes in RPMI-1640 containing $10 \%$ FBS. After reaching $50 \%$ confluency, the medium was replaced by fresh RPMI-1640 containing $10 \%$ FCS, and transfection with siRNA (si-Ctrl and si-CTGF) was performed using Lipofectamine RNAiMAX Transfection Regent. Forty-eight hours after transfection, the cells were trypsinized and pelleted. Subsequently, the cells were re-plated in the upper chambers of Transwell plates at a density of $1.0 \times 10^{6} / \mathrm{ml}$ in $200 \mu \mathrm{l}$ of RPMI-1640. The lower chamber contained $700 \mu \mathrm{l}$ of RPMI-1640 supplemented with $10 \%$ FBS. The subsequent procedure was the same as described above. We performed four individual experiments, and each assay was performed in triplicate.

Western blot analysis. The western blot experimental procedure was described previously (24). The following primary antibodies were used: Anti-E-cadherin (cat. no. 3195, at a 1:1,000 dilution; Cell Signaling Technology), anti-fibronectin (cat. no. sc-18825, at a 1:1,000 dilution; Santa Cruz Biotechnology, Inc.), anti-vimentin (cat. no. 5741, at a 1:1,000 dilution; Cell Signaling Technology), and anti-CTGF (cat. no. sc-365970, at a 1:1,000 dilution; Santa Cruz Biotechnology, Inc.). The primary antibodies were washed in $0.05 \%$ Tween-20/PBS and then incubated with horseradish peroxidase-conjugated secondary antibody. Proteins were visualized using Amersham ELC Western Blotting Detection Reagent (GE Healthcare Life Sciences). Bands were visualized using ImageQuant LAS 4000 mini (GE Healthcare Life Sciences).

$R N A$ extraction and quantitative real-time PCR. The procedure was previously described $(25,26)$. Total RNA was isolated using RNeasy Mini (Qiagen, Hilden, Germany). The concentration of RNA was measured according to absorbance at $260 \mathrm{~nm}$, and $0.5 \mathrm{mg}$ of total RNA was reverse-transcribed using M-MLV reverse transcriptase and a random primer using ReverTra Ace qPCR RT Master Mix (Toyobo, Osaka, Japan) at $42^{\circ} \mathrm{C}$ for $60 \mathrm{~min}$. Then, $1 \mu \mathrm{l}$ of the final cDNA solution was subjected to PCR using KOD DNA polymerase (Toyobo). Amplification conditions were as follows: Denaturation at $98^{\circ} \mathrm{C}$ for $2 \mathrm{~min}$; 40 cycles at $98^{\circ} \mathrm{C}$ for $10 \mathrm{sec}, 55^{\circ} \mathrm{C}$ for $10 \mathrm{sec}$, and $68^{\circ} \mathrm{C}$ for $30 \mathrm{sec}$ using LightCycler Nano (Roche Diagnostics, Basel, Switzerland). GAPDH was used as an endogenous control, relative expression was estimated using the comparative $\mathrm{Cq}\left(2^{-\Delta \Delta \mathrm{Cq}}\right)$ method. The sequences of primers used for the experiments were as follows: CTGF forward, 5'-TTCCAGAGCAGCTGC AAGTA and reverse, 5'-GCCAAACGTGTCTTCCAGTC.

Cytokine stimulation. EOC cell lines were stimulated with recombinant human TGF- $\beta 1(10 \mathrm{ng} / \mathrm{ml})$ (PeproTech, Inc., Rocky Hill, NJ, USA), hepatocyte growth factor (HGF) (40 ng/ml) (PeproTech, Inc.) and tumor necrosis factor $\alpha$ (TNF- $\alpha)(100 \mathrm{ng} / \mathrm{ml})$ (R\&D System, Minneapolis, MN, USA) in RPMI-1640 medium supplemented with 10\% FBS for each incubation time.

Patients and immunohistochemical staining. A total of 104 human EOC tissues were obtained from patients who underwent surgical treatment at Nagoya University Hospital between January 1994 and December 2010 after providing informed consent. In regards to the histological types, we 
adopted the World Health Organization (WHO) classification criteria (27). The clinical stage was assigned according to the International Federation of Gynecology and Obstetrics (FIGO) staging system $(28,29)$. This study was approved by the Ethics Committee of Nagoya University (Approval No. 2011-1234-2).

Formalin-fixed, paraffin-embedded tissue sections were cut at a thickness of $4 \mu \mathrm{m}$. For heat-induced epitope retrieval, deparaffinized sections in $0.01 \mathrm{M}$ citrate buffer were heated three times at $90^{\circ} \mathrm{C}$ for $5 \mathrm{~min}$ using a microwave oven. Sections were incubated at $4^{\circ} \mathrm{C}$ for $12 \mathrm{~h}$ with primary antibody (anti-goat-CTGF polyclonal, sc-14939, at a 1:100 dilution; Santa Cruz Biotechnology, Inc.). The sections were rinsed and incubated for $30 \mathrm{~min}$ with biotinylated anti-goat $\mathrm{IgG}$ antibody (Histofine SAB-PO (goat) kit, cat. no. 414012; Nichirei Corp., Tokyo, Japan). The immunoreactive staining was processed using the peroxidase-anti-peroxidase method according to the manufacturer's instructions (Dako, Hamburg, Germany). To detect the reaction, 3,3'-diaminobenzidine tetrachloride (DAB) chromogen solution was used. After rinsing in water for $30 \mathrm{~min}$, the sections were counterstained with hematoxylin and then dehydrated. Finally, they were mounted in mounting medium for examination.

Evaluation of immunohistochemical staining. For evaluation of the results of immunohistochemical staining, 10 fields for each specimen were selected and evaluated with both low- (x100) and high- (x400) power microscopy (Axio Imager A1, Carl Zeiss). Two investigators assessed the slides without knowledge of the clinicopathologic features and were blinded to each other's evaluation. The two investigators were in agreement on all the slides examined. Based on the immunostaining activity, a semiquantitative score was assigned according to the intensity and area of the stained cells, as described previously (30). For the evaluation of CTGF expression, the staining intensity was scored as 0 (negative-weak), 1 (medium), 2 (strong), or 3 (very strong). The percentage of the staining area was scored as $0(0-10 \%), 1(11-50 \%)$, and $2(51-100 \%)$ relative to the total tumor area. The sum of the staining intensity and area scores was calculated as the final score (0-5) for CTGF. Tumors with final scores of $0-1$ and 2-5 were classified as showing low and high expression, respectively.

Survival analyses. The distributions of the clinicopathologic factors were assessed using the Chi-square test. The progression-free survival (PFS) was defined as the time interval between the date of surgery and date of the last follow-up or recurrence/progression. The overall survival (OS) was defined as the time interval between the date of surgery and date of the last follow-up or death from any cause. The survival curves were compared employing the Log-rank test. Survival analysis was conducted using the Kaplan-Meier method. The prognostic significance of CTGF expression concerning other clinicopathologic variables was assessed using univariable and multivariable Cox's proportional hazard's analyses. All statistical analyses were performed with JMP Pro version 10.0 (SAS Institute, Japan). A P-value of $<0.05$ was considered indicative of statistical significance.

Statistical analysis. All data are expressed as the mean \pm SD. Data were calculated from at least three independent experiments. The significance of differences was analyzed by Student's t-test or one-way analysis of variance (ANOVA) followed by Tukey's post-hoc test. A P-value of $<0.05$ was considered indicative of statistical significance.

\section{Results}

Patient characteristics. We initially examined CTGF expression and its possible association with the prognostic outcome of EOC patients employing an immunohistochemical experiment. Patient characteristics are presented in Table I. The median age was 54, ranging from 24-78 years. The distributions of the FIGO stages were: $42.3 \%$ (44/104) stage I, 19.2\% (20/104) stage II, $34.6 \%$ (36/104) stage III and 3.8\% (4/104) stage IV. Among all patients, pathological types were as follows: 34 (32.7\%) with serous, 42 (40.4\%) with clear-cell, 18 (17.3\%) with endometrioid, and $8(7.7 \%)$ with mucinous carcinoma. One hundred and one $(97.1 \%)$ patients underwent postoperative chemotherapy. Three patients did not receive postoperative chemotherapy due to their severe complications or strong wishes. Among the 104 patients, lower- and higher-level CTGF staining expressions were noted in $65(62.5 \%)$ and 39 (37.5\%) patients, respectively. Representative images of various immunohistochemical intensities are presented in Fig. 1. The distributions of the age, stage, histological type, chemotherapy, and period of initial treatment did not differ between the two groups Table I.

Clinical outcome based on CTGF expression. The median (range) follow-up duration was 93.6 (3.8-284.4) months in all patients. During the observation period, 48 (46.1\%) patients experienced recurrence and 34 (32.7\%) succumbed to the disease. The median times to recurrence and death were 17.2 and 26.0 months, respectively. The five-year PFS and OS rates of all patients were 57.4 and $75.1 \%$, respectively. Patients in the higher-level CTGF group showed poorer PFS and OS rates than those in the lower-level group [PFS (log-rank: $\mathrm{P}=0.0076$, and OS (log-rank: $\mathrm{P}=0.0078$ ), respectively] (Fig. 2A and $\mathrm{B}$ ). Analysis of those with clear-cell carcinoma (CCC) revealed no significant difference in PFS or OS between the two groups (Fig. 3A and B). In contrast, analysis of those with a non-clear-cell (non-CCC) histology revealed that CTGF expression was significantly correlated with poorer long-term survival (PFS: $\mathrm{P}=0.0036$, OS: $\mathrm{P}=0.0070$ ) (Fig. 3C and D).

Univariate and multivariate analyses. We next conducted univariate and multivariate Cox proportional analyses regarding PFS/OS, FIGO stage (I vs. II-IV), age ( $\leq 50$ vs. $>50$ years), histological type (non-clear-cell vs. clear-cell), chemotherapy (taxane plus platinum vs. other chemotherapy or none), period of initial treatment (before 2005 vs. after 2006), and CTGF immunoreactivity (low vs. high) (Table SI). Based on univariate analysis, the FIGO stage, histological type, and CTGF expression were significant prognostic indicators of a poorer PFS. We evaluated the age, stage, histological type and CTGF expressions in multivariate analysis. This analysis demonstrated that the CTGF expression was a significantly independent predictor of a poorer OS and PFS [OS: Hazard ratio (HR): $2.141,95 \%$ confidence interval (CI): 1.077-4.296 $(\mathrm{P}=0.0300)$; PFS: HR (high vs. low): $1.837,95 \%$ CI: 1.023-3.289 $(\mathrm{P}=0.0418)]$. 
Table I. Association between the expression of CTGF and clinicopathologic parameters of the EOC cases.

\begin{tabular}{|c|c|c|c|c|c|c|}
\hline \multirow[b]{3}{*}{ Characteristics } & \multicolumn{5}{|c|}{ CTGF expression } & \multirow[b]{3}{*}{ P-value } \\
\hline & \multirow{2}{*}{$\frac{\text { Total }}{\mathrm{N}}$} & \multicolumn{2}{|c|}{ Low } & \multicolumn{2}{|c|}{ High } & \\
\hline & & $\mathrm{N}$ & $\%$ & $\mathrm{~N}$ & $\%$ & \\
\hline Total & 104 & 65 & 62.5 & 39 & 37.5 & \\
\hline Age (years) & & & & & & 0.4617 \\
\hline$\leq 50$ & 38 & 22 & 33.8 & 16 & 41.0 & \\
\hline$>50$ & 66 & 43 & 66.2 & 23 & 59.0 & \\
\hline FIGO stage & & & & & & 0.138 \\
\hline I & 44 & 31 & 47.7 & 13 & 33.3 & \\
\hline II & 20 & 14 & 21.5 & 6 & 15.4 & \\
\hline III & 36 & 17 & 26.2 & 19 & 48.7 & \\
\hline IV & 4 & 3 & 4.6 & 1 & 2.6 & \\
\hline Histological type (WHO) & & & & & & 0.2283 \\
\hline Serous & 34 & 18 & 27.7 & 16 & 41.0 & \\
\hline Clear-cell & 42 & 27 & 41.5 & 15 & 38.5 & \\
\hline Endometroid & 18 & 12 & 18.5 & 6 & 15.4 & \\
\hline Mucinous & 8 & 7 & 10.8 & 1 & 2.6 & \\
\hline Mixed type & 1 & 1 & 1.5 & 0 & 0.0 & \\
\hline Adenocarcinoma & 1 & 0 & 0.0 & 1 & 2.6 & \\
\hline Chemotherapy & & & & & & 0.3367 \\
\hline None & 3 & 3 & 4.6 & 0 & 0.0 & \\
\hline Platinum-based & 7 & 5 & 7.7 & 2 & 5.1 & \\
\hline Taxane plus platinum & 94 & 57 & 87.7 & 37 & 94.9 & \\
\hline Period of initial treatment & & & & & & 0.5496 \\
\hline Before 2005 & 33 & 22 & 33.8 & 11 & 28.2 & \\
\hline After 2006 & 71 & 43 & 66.2 & 28 & 71.8 & \\
\hline
\end{tabular}

EOC, epithelial ovarian carcinoma; CTGF, connective tissue growth factor; FIGO, International Federation of Gynecology and Obstetrics.
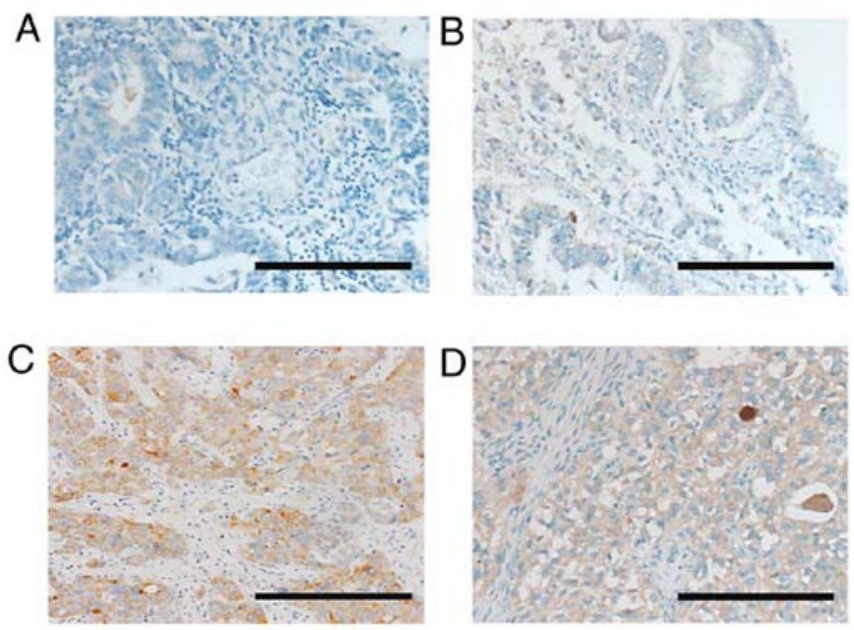

Figure 1. Immunoreactive CTGF expression in ovarian EOC tissues (paraffin sections). Based on the CTGF immunostaining activity, a semiquantitative score was assigned according to the intensity and area of stained cells, as described in Materials and methods. (A) Negative-weak (score 0: Low), (B) medium (score 1: Low), (C) strong (score 3: High), (D) very strong (score 5: High). Magnification x100; scale bars, $50 \mu \mathrm{m}$. EOC, epithelial ovarian carcinoma; CTGF, connective tissue growth factor.
Involvement of CTGF expression in the tumor-promoting effect. Subsequently, we examined the role of CTGF in the malignant properties of EOC in vitro. Previously, we generated PTX-resistant cell lines using parental EOC cells (NOS2). We treated these cells for months with stepwisely increasing concentrations of PTX and finally generated highly PTX-resistant NOS2TR cells (Fig. 4A). The NOS2TR cells displayed a spindle-shaped morphology with looser cell-cell adhesion. Transforming growth factor (TGF)- $\beta$ as well as hepatocyte growth factor (HGF) and tumor necrosis factor (TNF)- $\alpha$ play an important role in the dissemination of ovarian cancer, stimulating tumor invasion and metastasis of tumor cells (31-33). When in the presence of TGF- $\beta$, the morphology of NOS2TR cells showed a more mesenchymal cell shape with decreased E-cadherin and increased vimentin expressions, compared with the parental NOS2 cells (Fig. 4B and C). Furthermore, the addition of TGF- $\beta$ to NOS2TR cells significantly increased the migratory potential compared with that noted in the parental NOS2 cells (Fig. 4D and E) $(\mathrm{P}<0.05)$.

We further explored the role of CTGF in the malignant properties of EOC using several in vitro experiments. We first investigated the expression of CTGF in various EOC cell 
A

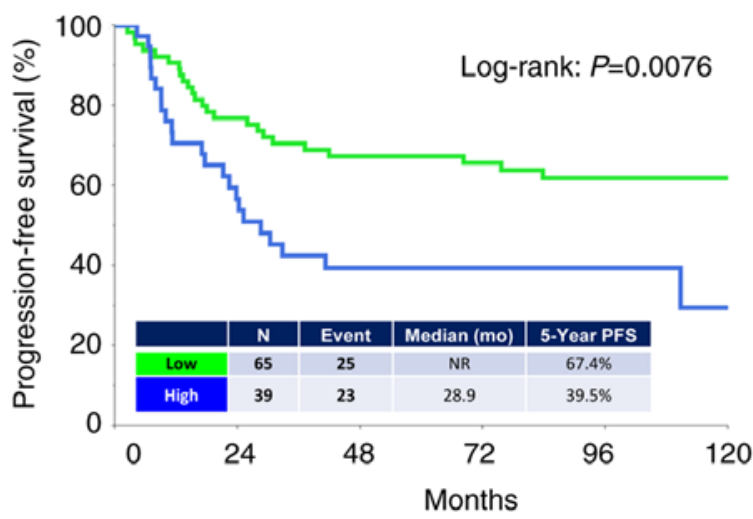

B

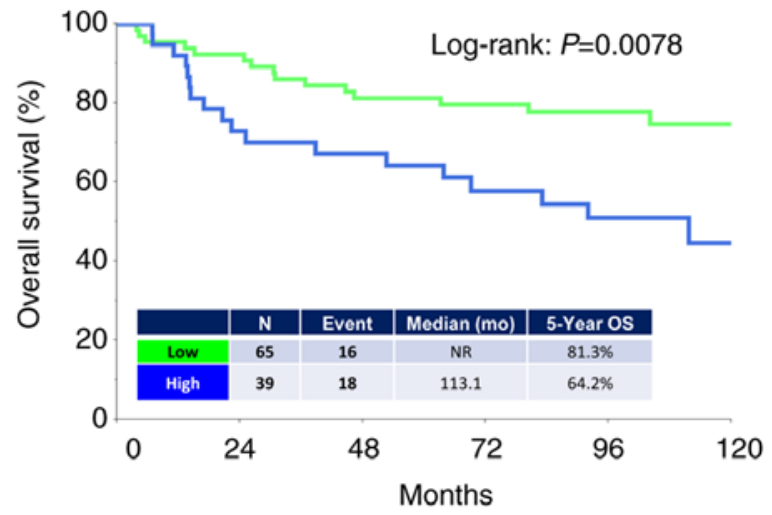

Figure 2. Kaplan-Meier progression-free survival (PFS) and overall survival (OS) curves of EOC patients according to the immunoreactivity of CTGF. (A) PFS. Green line indicates low CTGF expression ( $\mathrm{N}=65)$. Blue line represents high CTGF immunoexpression $(\mathrm{N}=39)$. Patients with positive CTGF expression showed a significantly poorer PFS ( $\mathrm{P}=0.0076)$. (B) OS. Green line indicates low CTGF expression $(\mathrm{N}=65)$. Blue line represents high CTGF immunoexpression $(\mathrm{N}=39)$. Patients positive for CTGF expression showed a significantly poorer OS ( $\mathrm{P}=0.0078)$. EOC, epithelial ovarian carcinoma; CTGF, connective tissue growth factor.
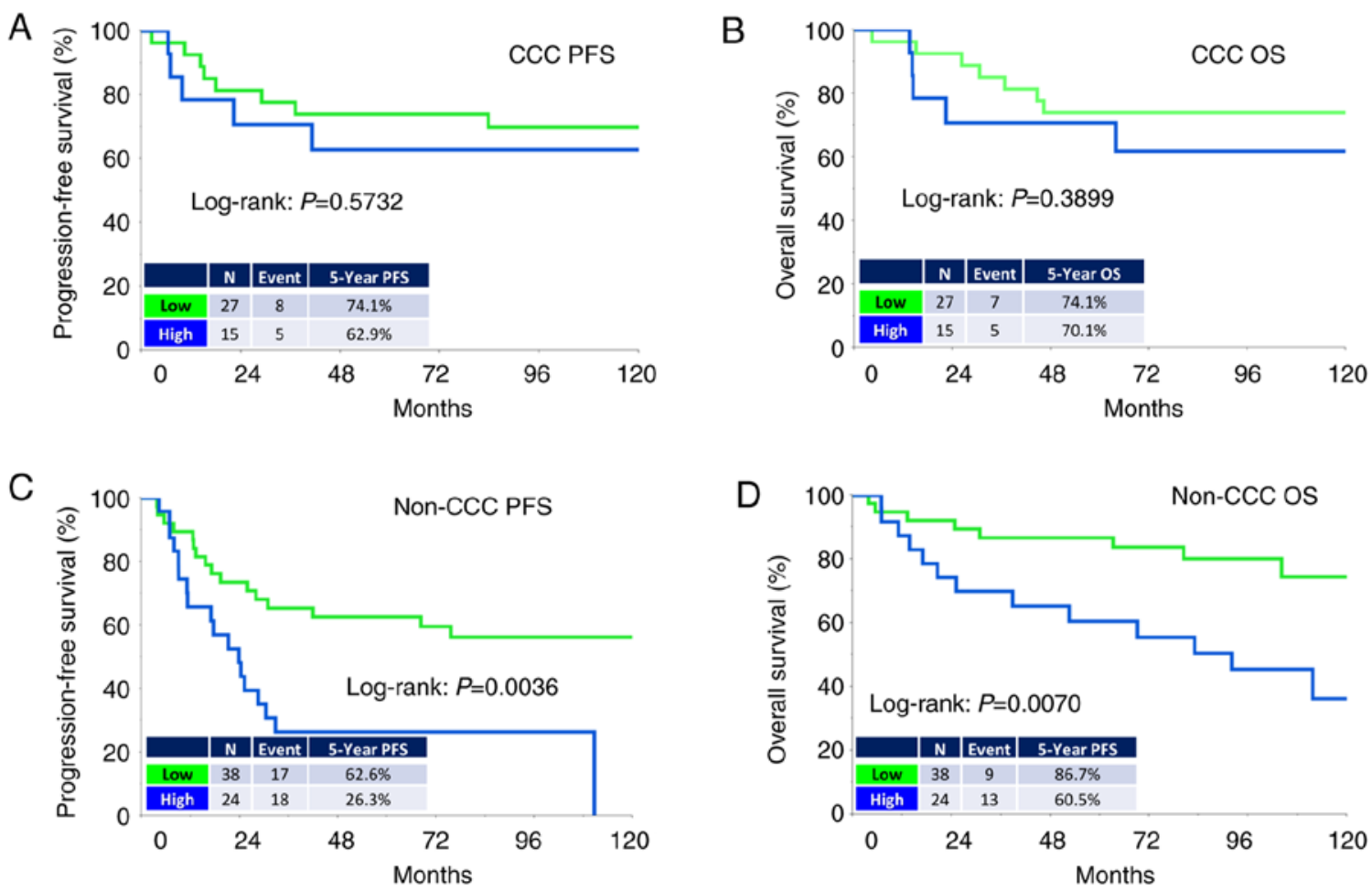

Figure 3. Kaplan-Meier progression-free survival (PFS) and overall survival (OS) curves of EOC patients with clear-cell carcinoma (CCC) or non-clear-cell carcinoma (Non-CCC) according to the immunoreactivity of CTGF. (A and B) CCC. Green line indicates low CTGF expression (N=27). Blue line represents high CTGF immunoexpression ( $\mathrm{N}=15)$. (C and D) Non-CCC. Green line indicates low CTGF expression $(\mathrm{N}=38)$. Blue line represents high $\mathrm{CTGF}$ immunoexpression ( $\mathrm{N}=24)$ [C (PFS): $\mathrm{P}=0.0036$ and $\mathrm{D}(\mathrm{OS}): \mathrm{P}=0.0070]$. $\mathrm{EOC}$, epithelial ovarian carcinoma; $\mathrm{CTGF}$, connective tissue growth factor.

lines. CTGF was expressed in ES-2 cells; however, a lower level of expression of CTGF was observed in the SKOV3, A2780, OVCAR, NOS2, and NOS2TR cells. The addition of TGF- $\beta$ induced the upregulation of CTGF expression in both the NOS2 and NOS2TR cell lines (Fig. 5A). However, the expression of CTGF following the addition of TGF- $\beta$ was more markedly increased in the NOS2TR than in the NOS2 cells. These results suggest that TGF- $\beta$ generated in the microenvironment through cell-to-cell communication may contribute to the enhancement of CTGF, leading to the acquisition of the chronic chemoresistance/metastatic potential of EOC. Thus, we next examined whether the acquired PTX-resistance of NOS2TR depended on the upregulation of CTGF. Using specific si-RNA of CTGF, we confirmed that the enhanced expression of CTGF in both cell lines was completely blocked at the protein and transcriptional levels (Fig. 5B and C). As shown in Fig. 5D, the enhanced migratory potential induced by the addition of TGF- $\beta$ was completely inhibited in the CTGF-depleted NOS2TR cells. In contrast, in control siRNA-transfected NOS2TR cells, we did not observe such an inhibitory effect (Fig. 5E). We subsequently investigated whether CTGF is associated with 

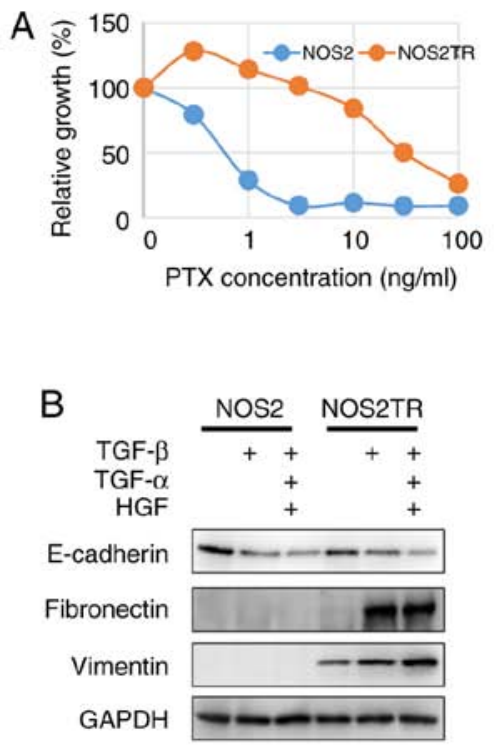

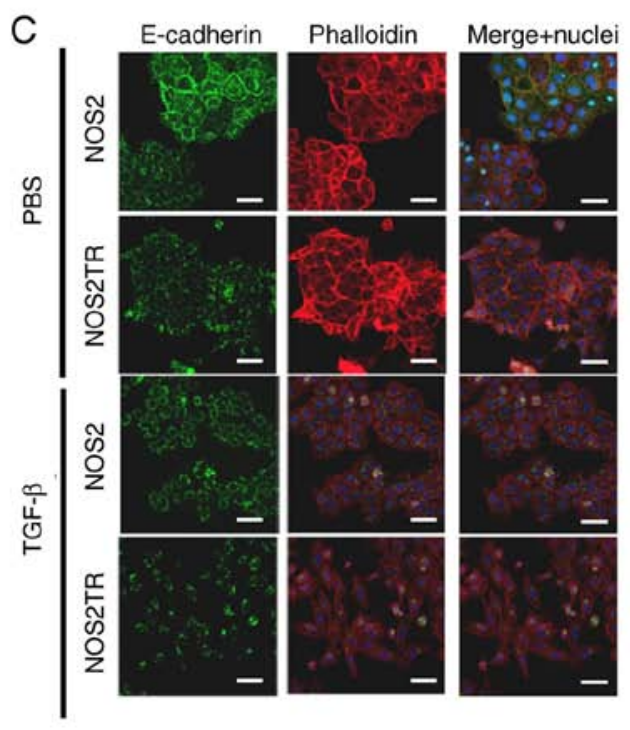

Figure 4. (A) Generation of two independent PTX-resistant cell lines using NOS2 cells by continuous exposure to stepwise-increasing concentrations of PTX. PTX sensitivity of the chronic PTX-resistant cells was assayed as described in Materials and methods. (B) Western blot analysis of E-cadherin, fibronectin and vimentin in the NOS2 and NOS2TR cells following treatment with TGF- $\beta(10 \mathrm{ng} / \mathrm{ml})$, TNF- $\alpha(100 \mathrm{ng} / \mathrm{ml})$ and HGF $(40 \mathrm{ng} / \mathrm{ml})$. (C) Immunofluorescence expression of E-cadherin in NOS2 and NOS2TR cells with or without TGF- $\beta(10 \mathrm{ng} / \mathrm{ml})$. Scale bars, $50 \mu \mathrm{m}$. (D) Images of the migration assay and quantitative analysis of the migration cell number. (E) "P<0.05 compared to the PBS control. PTX, paclitaxel; TGF- $\beta$, transforming growth factor- $\beta$; HGF, hepatocyte growth factor; TNF- $\alpha$, tumor necrosis factor- $\alpha$.
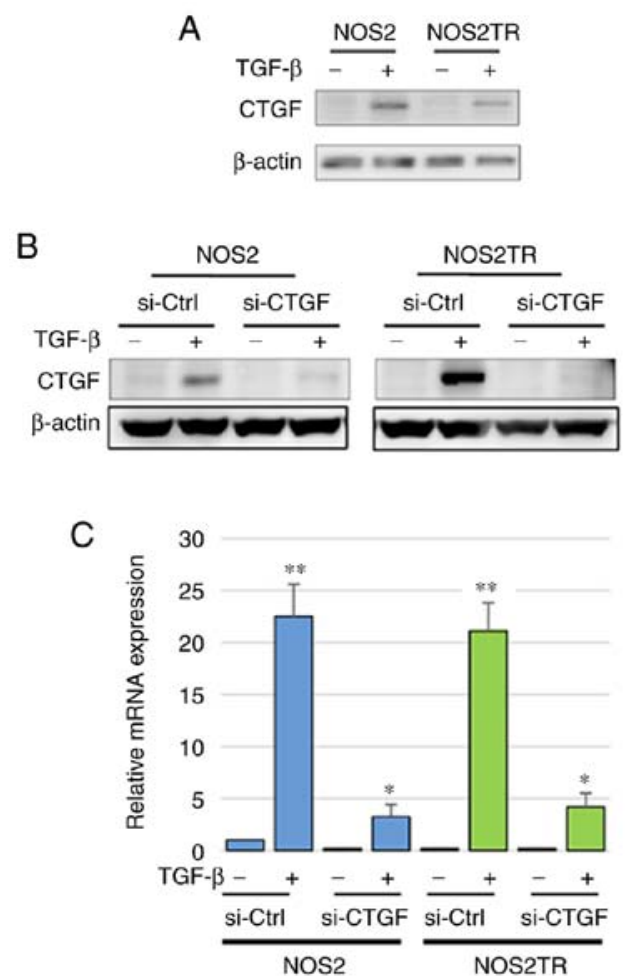
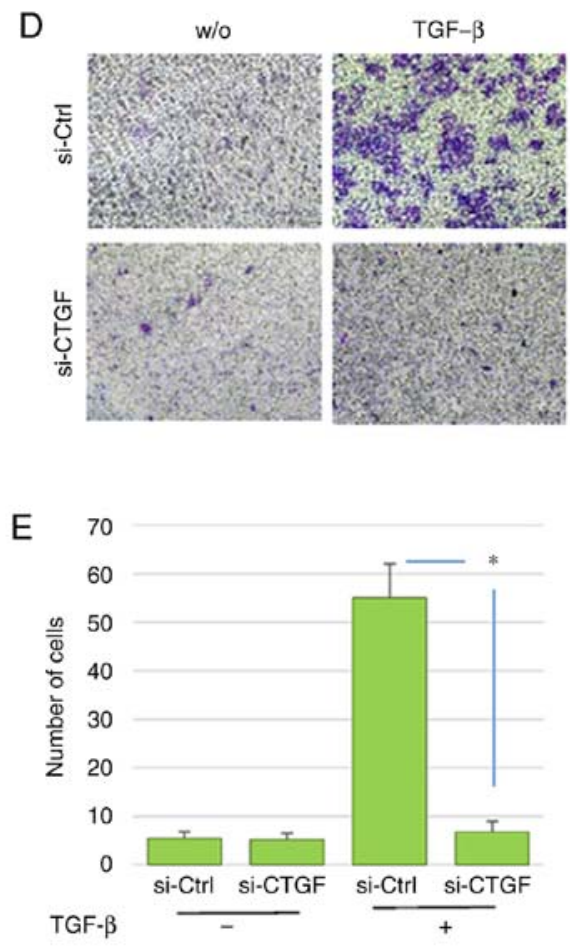

Figure 5. (A) Western blot analysis of the protein expression of CTGF in NOS2 and NOS2TR cells with or without TGF- $\beta$ (10 ng/ml). The addition of TGF- $\beta$ resulted in increased CTGF expression. (B) The protein expression of CTGF in NOS 2 and NOS2TR cells. The TGF- $\beta$-induced CTGF expression was completely inhibited in CTGF-depleted NOS2 and NOS2TR cells compared with that of the control siRNA-transfected cells. (C) qPCR of CTGF mRNA expression. ${ }^{*} \mathrm{P}<0.05,{ }^{* *} \mathrm{P}<0.01$, significant difference compared to the si-Ctrl group. (D) The migratory ability of the si-CTGF or si-Ctrl transfected NOS2TR cells in the presence or absence of TGF- $\beta$ (10 ng/ml). (E) The number of migrating cells. * $\mathrm{P}<0.05$, significant difference compared with the si-Ctrl group. CTGF, connective tissue growth factor; TGF- $\beta$, transforming growth factor- $\beta$.

the chemoresistance-promoting effect in vitro. The NOS3TR cells, another type of chronic PTX-resistant EOC cell line, transfected with siRNAs (si-CTGF) were assessed by the
PTX-sensitivity assay as described above. In NOS3TR cells, the silencing of CTGF expression led to restoration of the PTX sensitivity (Fig. 6A and B). Furthermore, we confirmed 
A
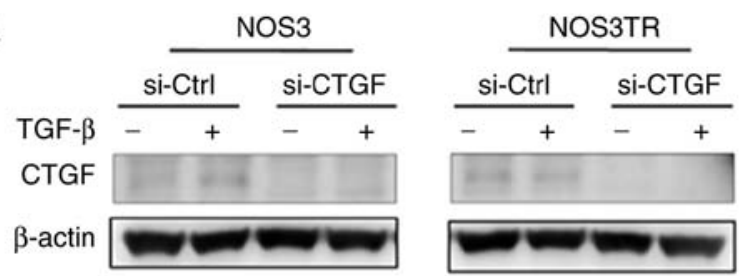

B

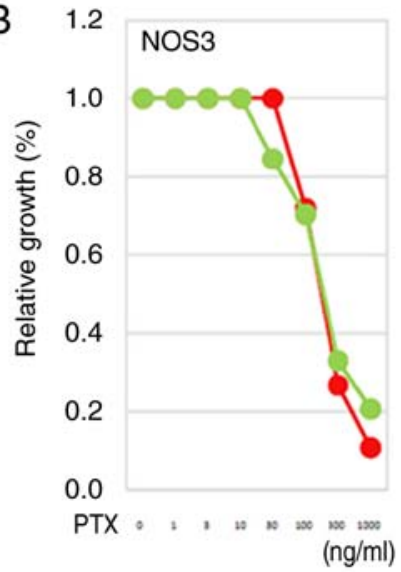

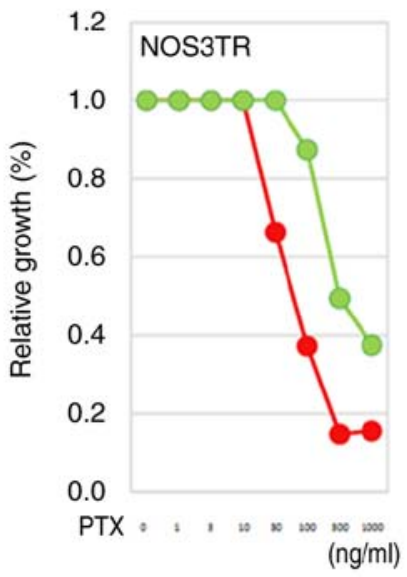

si-Ctrl
C
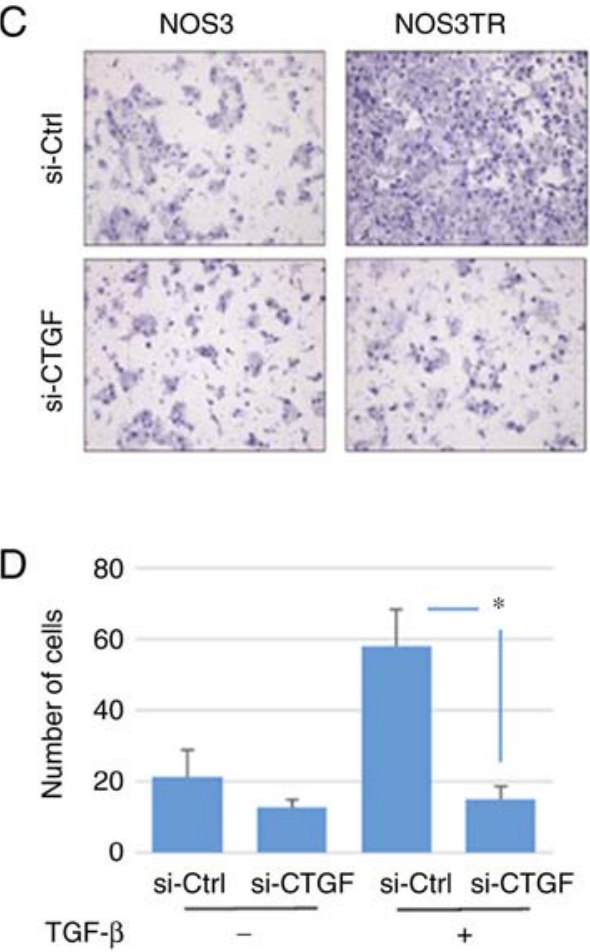

Figure 6. (A) Protein expression of CTGF in NOS3 and NOS3TR cells with or without TGF- $\beta$. (B) Effect of the silencing of CTGF expression on PTX-chemosensitivity in NOS3 and NOS3TR cells. (C) The migratory ability of the si-CTGF or si-Ctrl transfected NOS3 or NOS3TR cells in the presence of TGF- $\beta$ (10 ng/ml). (D) The number of migrated cells. ${ }^{*} \mathrm{P}<0.05$, significant difference compared with the si-Ctrl group. CTGF, connective tissue growth factor; PTX, paclitaxel; TGF- $\beta$, transforming growth factor- $\beta$.

that the TGF- $\beta$-dependent migration-promoting effect was completely inhibited in CTGF-depleted NOS3TR cells, as observed in NOS3 cells (Fig. 6C and D) $(\mathrm{P}=0.05)$.

\section{Discussion}

In the present study, we initially revealed that connective tissue growth factor (CTGF) expression was significantly correlated with a poorer prognostic outcome in epithelial ovarian carcinoma (EOC) patients. Indeed, those with higher-level CTGF expression demonstrated poorer OS and PFS rates than those with lower-level expression. Moreover, multivariate analyses showed that higher-level CTGF expression was an independent prognostic indicator of poorer survival in those cases. Various studies have revealed positive associations among CTGF expression, aggressive features, and a poor oncologic outcome in patients with a number of solid and hematological malignancies, including pre-B acute lymphoblastic leukemia, gallbladder cancer, pancreatic carcinoma and esophageal squamous cell carcinoma (34-37). Wang et al demonstrated that CTGF expression was negatively associated with the response to chemotherapy in breast cancer patients who received neoadjuvant chemotherapy, and its cellular overexpression resulted in resistance to doxorubicin- and paclitaxel-induced apoptosis by the upregulation of Bcl-xL and cellular inhibitor of apoptosis protein 1 (cIAP1) (38). Mao et al reported that the expression of CTGF was significantly upregulated in clinical tissues of gastric carcinoma patients, and the overexpression of CTGF in gastric carcinoma cells promoted their migratory capability in vitro and significantly increased tumor metastasis in nude mice (39). Furthermore, the downregulation of pancreatic tumor cells was found to lead to markedly reduced growth on soft agar and in a murine subcutaneous implantation model, and CTGF expression and secretion were found to be increased in hypoxic pancreatic tumors (35). The current findings are consistent with these results. However, in the present study, although patients with a non-clear-cell histology and similar prognostic tendencies were observed, analysis of those with clear-cell carcinoma (CCC) revealed no significant difference between the low and high CTGF expression groups. Indeed, EOC consists of heterogeneous histological types with a different tumor biology. CCC patients in general display a lower response rate to platinum-based compounds, leading to intrinsic chemoresistance $(40,41)$. Thus, it is likely that chemoresistance of this tumor is based on a CTGF-independent mechanism. Indeed, earlier studies did not refer to the effect of CTGF expression on a variety of histological types of EOC tumors. In a future study, this observation should be verified.

A possible explanation of the poor survival in patients with higher-level CTGF expression may be the enhanced metastatic potential and chronic chemoresistance of this tumor. Our previous study revealed that chronic PTX resistance induced more marked mesenchymal hallmarks, including the switch of an epithelial to a fibroblast-like morphology, upregulation of epithelial-mesenchymal-transition-related biomarkers, and the enhancement of motile capabilities (20). In the present study, the silencing of CTGF significantly restored PTX susceptibility and reduced invasiveness/motility in cells 
chronically resistant to PTX. This suggests that the development/maintenance of both the PTX-resistance and metastatic ability of these cells were attributable to CTGF expression. A previous study showed that the expression of CTGF was upregulated in human osteosarcoma cells after treatment with cisplatin, and CTGF overexpression induced enhanced resistance to cisplatin-mediated cell apoptosis through upregulations of $\mathrm{Bcl}-\mathrm{xL}$ and surviving (16). Yang et al demonstrated that the overexpression of CTGF enhanced resistance to 5-FU-induced cell apoptosis. They also reported that downregulation of the expression of CTGF promoted the curative effect of chemotherapy and blocked the cell cycle in the G1 phase (15). Moreover, the expression of CTGF was found to be associated with increased resistance to PTX-mediated cell apoptosis through the upregulation of survivin and the AMP-activated protein kinase (AMPK)-dependent nuclear factor $\kappa B$ pathway (42). In the treatment of EOC patients, acquired or intrinsic chemoresistance is a major clinical cause of a poor prognosis. The present study demonstrated that CTGF plays a central role in the chemoresistance of EOC cells.

As well as tumor cells, stromal-cell derived CTGF expression can exert tumor-promoting effects, such as proliferation and invasion in glioma as well as pancreatic and prostate cancer (43-45). Yang et al demonstrated that stromal expression of CTGF induced significant increases in microvessel density and xenograft tumor growth, suggesting that this molecule is one of the key regulators of angiogenesis in the tumor-reactive stromal microenvironment as a downstream mediator of TGF- $\beta 1$ (45). Similarly, according to a recent study by Kim et al, immunohistochemical analyses of high-grade serous ovarian tumors revealed that the highest level of tumor stromal CTGF expression was correlated with the poorest prognosis (46). Based on the molecular signature in a subset of high-grade serous ovarian cancer (HGSOC) samples that was primarily driven by a high stromal response, CTGF was overexpressed in the stroma of these tumors in association with a poor oncologic outcome (47). In our current analyses, we assessed the CTGF immunoactivity in tumorous tissues. Indeed, CTGF may function in the stromal cells as a metastasis-promoting factor through tumor-stromal interaction. Considering the CTGF expression as a molecular target of epithelial ovarian cancer (EOC) with aggressive behavior, this substance plays an important role in not only stromal-cancer communication but also the multidisciplinary functions of tumor cells, including the development of chemoresistance and metastatic potential.

The TGF- $\beta$ signaling pathways are crucial regulators of the multiple steps of the tumor microenvironment associated with EMT (48-50). TGF- $\beta$ was found to lead to a long-lasting upregulation of CTGF mRNA and protein expression in mouse and human proximal tubular epithelial cell lines (51). Tsai et al reported that CTGF is an essential downstream mediator of TGF- $\beta 1$-induced extracellular matrix production and myofibroblast transdifferentiation in Graves' orbital fibroblasts (52). Our previous study demonstrated that TGF- $\beta$ is generated by EOC cells, which was synergistically upregulated under co-culture conditions with human mesothelial cells (23). In the present study, it was demonstrated that TGF- $\beta$ stimulation led to an increase in CTGF expression. Furthermore, it was revealed that TGF- $\beta$-induced migratory potential and restoration of PTX sensitivity were completely inhibited in CTGF-silenced PTX-chemoresistant cells. Thus, there may be a close link between the enhanced metastatic potential and PTX resistance of EOC via the TGF- $\beta / C T G F$ axis. However, in the present study, we did not conduct relevant experiments regarding CTGF overexpression. We consider that this was one of the critical limitations of the present investigation. We hope to verify the significance of CTGF overexpression in a future investigation. Overall, we consider CTGF to be a valuable biomarker and effective therapeutic target for EOC. Therefore, CTGF may be a therapeutic candidate for modulating the PTX sensitivity of EOC.

In summary, we identified CTGF as a prognostic indicator of and therapeutic target for EOC, particularly in non-clear-cell carcinoma. An unfavorable outcome in patients with higher-level CTGF expression may be due to the increased metastatic capability and chemoresistance of EOC. However, the detailed functions of CTGF remain unclear. We hope that the further mechanistic elucidation of CTGF will contribute to improving treatment for EOC patients by adding criteria for the administration of systematic therapy in the future.

\section{Acknowledgements}

We sincerely thank Mrs. M. Sugiyama (research technician at Nagoya University, Graduate School of Medicine) for advice on the in vitro experiments.

\section{Funding}

This study was supported by JSPS (Japan Society for the Promotion of Science) KAKENHI Grants-in-Aid for Scientific Research (grant nos. 17H04338 and 16K15704).

\section{Availability of data and materials}

The datasets used during the present study are available from the corresponding author upon reasonable request.

\section{Authors' contributions}

AS and HK carried out the data analysis and interpretation and writing of the manuscript. ST, SS, YI, KN, JS, KN and NY carried out the data collection. FK supervised the research and was responsible for the funding. All authors read and approved the manuscript and agree to be accountable for all aspects of the research in ensuring that the accuracy or integrity of any part of the work are appropriately investigated and resolved.

\section{Ethics approval and consent to participate}

The present study was approved by the Ethics Committee of Nagoya University (Approval No. 2011-1234-2).

\section{Patient consent for publication}

Not applicable. 


\section{Competing interests}

All authors declare that there are no competing interests, and no financial or personal relationships with other people or organizations that could inappropriately influence this work.

\section{References}

1. Bray F, Ferlay J, Soerjomataram I, Siegel RL, Torre LA and Jemal A: Global cancer statistics 2018: GLOBOCAN estimates of incidence and mortality worldwide for 36 cancers in 185 countries. CA Cancer J Clin 68: 394-424, 2018.

2. Brun JL, Feyler A, Chene G, Saurel J, Brun G and Hocke C: Long-term results and prognostic factors in patients with epithelial ovarian cancer. Gynecol Oncol 78: 21-27, 2000.

3. Thiery JP: Epithelial-mesenchymal transitions in tumour progression. Nat Rev Cancer 2: 442-454, 2002.

4. Boyer B, Valles AM and Edme N: Induction and regulation of epithelial-mesenchymal transitions. Biochem Pharmacol 60: 1091-1099, 2000

5. Vernon $\mathrm{AE}$ and LaBonne C: Tumor metastasis: A new twist on epithelial-mesenchymal transitions. Curr Biol 14: R719-R721, 2004.

6. Eisenkop SM, Friedman RL and Wang HJ: Complete cytoreductive surgery is feasible and maximizes survival in patients with advanced epithelial ovarian cancer: A prospective study. Gynecol Oncol 69: 103-108, 1998.

7. Kajiyama H, Mizuno M, Shibata K, Kawai M, Nagasaka T and Kikkawa F: Extremely poor postrecurrence oncological outcome for patients with recurrent mucinous ovarian cancer. Int J Clin Oncol 19: 121-126, 2014.

8. Kajiyama H, Shibata K, Mizuno M, Umezu T, Suzuki S, Yamamoto E, Fujiwara S, Kawai M, Nagasaka T and Kikkawa F: Long-term clinical outcome of patients with recurrent epithelial ovarian carcinoma: Is it the same for each histological type? Int J Gynecol Cancer 22: 394-399, 2012.

9. Brigstock DR, Goldschmeding R, Katsube KI, Lam SC, Lau LF, Lyons K, Naus C, Perbal B, Riser B, Takigawa M and Yeger H: Proposal for a unified CCN nomenclature. Mol Pathol 56: 127-128, 2003

10. Brigstock DR: The connective tissue growth factor/cysteine-rich $61 /$ nephroblastoma overexpressed (CCN) family. Endocr Rev 20: 189-206, 1999.

11. Leask A and Abraham DJ: All in the CCN family: Essential matricellular signaling modulators emerge from the bunker. J Cell Sci 119: 4803-4810, 2006.

12. Tsai HC, Su HL, Huang CY, Fong YC, Hsu CJ and Tang CH: CTGF increases matrix metalloproteinases expression and subsequently promotes tumor metastasis in human osteosarcoma through down-regulating miR-519d. Oncotarget 5: 3800-3812, 2014.

13. Han Q, Zhang HY, Zhong BL, Wang XJ, Zhang B and Chen H: MicroRNA-145 inhibits cell migration and invasion and regulates epithelial-mesenchymal transition (EMT) by targeting connective tissue growth factor (CTGF) in esophageal squamous cell carcinoma. Med Sci Monit 22: 3925-3934, 2016.

14. Lai D, Ho KC, Hao Y and Yang X: Taxol resistance in breast cancer cells is mediated by the hippo pathway component TAZ and its downstream transcriptional targets Cyr61 and CTGF. Cancer Res 71: 2728-2738, 2011.

15. Yang K, Gao K, Hu G, Wen Y, Lin C and Li X: CTGF enhances resistance to 5-FU-mediating cell apoptosis through FAK/MEK/ERK signal pathway in colorectal cancer. Onco Targets Ther 9: 7285-7295, 2016.

16. Tsai HC, Huang CY, Su HL and Tang CH: CCN2 enhances resistance to cisplatin-mediating cell apoptosis in human osteosarcoma. PLoS One 9: e90159, 2014.

17. Wang L, He J, Xu H, Xu L and Li N: MiR-143 targets CTGF and exerts tumor-suppressing functions in epithelial ovarian cancer. Am J Transl Res 8: 2716-2726, 2016.

18. Kajiyama H, Kikkawa F, Suzuki T, Shibata K, Ino K and Mizutani S: Prolonged survival and decreased invasive activity attributable to dipeptidyl peptidase IV overexpression in ovarian carcinoma. Cancer Res 62: 2753-2757, 2002.

19. Kajiyama H, Shibata K, Ino K, Mizutani S, Nawa A and Kikkawa F: The expression of dipeptidyl peptidase IV (DPPIV/CD26) is associated with enhanced chemosensitivity to paclitaxel in epithelial ovarian carcinoma cells. Cancer Sci 101: $347-354,2010$
20. Kajiyama H, Shibata K, Terauchi M, Yamashita M, Ino K, Nawa A and Kikkawa F: Chemoresistance to paclitaxel induces epithelial-mesenchymal transition and enhances metastatic potential for epithelial ovarian carcinoma cells. Int J Oncol 31: 277-283, 2007.

21. Maeda O, Shibata K, Hosono S, Fujiwara S, Kajiyama H, Ino K, Nawa A, Tamakoshi K and Kikkawa F: Spectrin $\alpha$ II and $\beta$ II tetramers contribute to platinum anticancer drug resistance in ovarian serous adenocarcinoma. Int J Cancer 130: 113-121, 2012.

22. Utsumi F, Kajiyama H, Nakamura K, Tanaka H, Mizuno M, Ishikawa K, Kondo H, Kano H, Hori M and Kikkawa F: Effect of indirect nonequilibrium atmospheric pressure plasma on anti-proliferative activity against chronic chemo-resistant ovarian cancer cells in vitro and in vivo. PLoS One 8: e81576, 2013.

23. Sakata J, Utsumi F, Suzuki S, Niimi K, Yamamoto E, Shibata K, Senga T, Kikkawa F and Kajiyama H: Inhibition of ZEB1 leads to inversion of metastatic characteristics and restoration of paclitaxel sensitivity of chronic chemoresistant ovarian carcinoma cells. Oncotarget 8: 99482-99494, 2017.

24. Hosono S, Kajiyama H, Terauchi M, Shibata K, Ino K, Nawa A and Kikkawa F: Expression of Twist increases the risk for recurrence and for poor survival in epithelial ovarian carcinoma patients. Br J Cancer 96: 314-320, 2007.

25. Livak KJ and Schmittgen TD. Analysis of relative gene expression data using real-time quantitative PCR and the 2(-Delta Delta C(T)) method. Methods 25: 402-408, 2001.

26. Sugiyama K, Kajiyama H, Shibata K, Yuan H, Kikkawa F and Senga T: Expression of the miR200 family of microRNAs in mesothelial cells suppresses the dissemination of ovarian cancer cells. Mol Cancer Ther 13: 2081-2091, 2014.

27. Kurman RJ, Carcangiu ML, Herrington CS and Young RH: WHO Classification of Tumours of Female Reproductive Organs. Fourth edition: IARC Press, 2014.

28. Zeppernick F and Meinhold-Heerlein I: The new FIGO staging system for ovarian, fallopian tube, and primary peritoneal cancer. Arch Gynecol Obstet 290: 839-842, 2014.

29. Chen VW, Ruiz B, Killeen JL, Coté TR, Wu XC and Correa CN: Pathology and classification of ovarian tumors. Cancer 97 (10 Suppl): S2631-S2642, 2003

30. Sakata J, Kajiyama H, Suzuki S, Utsumi F, Niimi K, Sekiya R, Shibata K, Senga T and Kikkawa F: Impact of positive ZEB1 expression in patients with epithelial ovarian carcinoma as an oncologic outcome-predicting indicator. Oncol Lett 14: 4287-4293, 2017.

31. Lau TS, Chan LK, Wong EC, Hui CW, Sneddon K, Cheung TH, Yim SF, Lee JH, Yeung CS, Chung TK and Kwong J: A loop of cancer-stroma-cancer interaction promotes peritoneal metastasis of ovarian cancer via TNF $\alpha-T G F \alpha-E G F R$. Oncogene 36: 3576-3587, 2017.

32. Nakamura M, Ono YJ, Kanemura M, Tanaka T, Hayashi M, Terai Y and Ohmichi M: Hepatocyte growth factor secreted by ovarian cancer cells stimulates peritoneal implantation via the mesothelial-mesenchymal transition of the peritoneum. Gynecol Oncol 139: 345-354, 2015

33. Wei W, Kong B, Yang Q and Qu X: Hepatocyte growth factor enhances ovarian cancer cell invasion through downregulation of thrombospondin-1. Cancer Biol Ther 9: 79-87, 2010.

34. Alvarez H, Corvalan A, Roa JC, Argani P, Murillo F, Edwards J, Beaty R, Feldmann G, Hong SM, Mullendore M, et al: Serial analysis of gene expression identifies connective tissue growth factor expression as a prognostic biomarker in gallbladder cancer. Clin Cancer Res 14: 2631-2638, 2008.

35. Bennewith KL, Huang X, Ham CM, Graves EE, Erler JT, Kambham N, Feazell J, Yang GP, Koong A and Giaccia AJ: The role of tumor cell-derived connective tissue growth factor (CTGF/CCN2) in pancreatic tumor growth. Cancer Res 69: 775-784, 2009

36. Boag JM, Beesley AH, Firth MJ, Freitas JR, Ford J, Brigstock DR, de Klerk NH and Kees UR: High expression of connective tissue growth factor in pre-B acute lymphoblastic leukaemia. Br J Haematol 138: 740-748, 2007.

37. Li LY, Li EM, Wu ZY, Huang X, Shen JH, Xu XE, Wu JY, Huang $\mathrm{Q}$ and $\mathrm{Xu} \mathrm{LY}$ : Connective tissue growth factor expression in precancerous lesions of human esophageal epithelium and prognostic significance in esophageal squamous cell carcinoma. Dis Esophagus 24: 337-345, 2011.

38. Wang MY, Chen PS, Prakash E, Hsu HC, Huang HY, Lin MT, Chang KJ and Kuo ML: Connective tissue growth factor confers drug resistance in breast cancer through concomitant up-regulation of Bcl-xL and cIAP1. Cancer Res 69: 3482-3491, 2009. 
39. Mao Z, Ma X, Rong Y, Cui L, Wang X, Wu W, Zhang J and Jin D: Connective tissue growth factor enhances the migration of gastric cancer through downregulation of E-cadherin via the NF-kB pathway. Cancer Sci 102: 104-110, 2011.

40. Sugiyama T, Kamura T, Kigawa J, Terakawa N, Kikuchi Y, Kita T, Suzuki M, Sato I and Taguchi K: Clinical characteristics of clear cell carcinoma of the ovary: A distinct histologic type with poor prognosis and resistance to platinum-based chemotherapy. Cancer 88: 2584-2589, 2000.

41. Shimada M, Kigawa J, Ohishi Y, Yasuda M, Suzuki M, Hiura M, Nishimura R, Tabata T, Sugiyama T and Kaku T: Clinicopathological characteristics of mucinous adenocarcinoma of the ovary. Gynecol Oncol 113: 331-334, 2009.

42. Tsai HC, Huang CY, Su HL and Tang CH: CTGF increases drug resistance to paclitaxel by upregulating survivin expression in human osteosarcoma cells. Biochim Biophys Acta 1843: 846-854, 2014.

43. Edwards LA, Woolard K, Son MJ, Li A, Lee J, Ene C, Mantey SA, Maric D, Song H, Belova G, et al: Effect of brainand tumor-derived connective tissue growth factor on glioma invasion. J Natl Cancer Inst 103: 1162-1178, 2011.

44. Eguchi D, Ikenaga N, Ohuchida K, Kozono S, Cui L, Fujiwara K, Fujino M, Ohtsuka T, Mizumoto K and Tanaka M: Hypoxia enhances the interaction between pancreatic stellate cells and cancer cells via increased secretion of connective tissue growth factor. J Surg Res 181: 225-233, 2013.

45. Yang F, Tuxhorn JA, Ressler SJ, McAlhany SJ, Dang TD and Rowley DR: Stromal expression of connective tissue growth factor promotes angiogenesis and prostate cancer tumorigenesis. Cancer Res 65: 8887-8895, 2005.

46. Kim HC, Ji W, Kim MY, Colby TV, Jang SJ, Lee CK, Han SB and Kim DS: Interstitial pneumonia related to undifferentiated connective tissue disease: Pathologic pattern and prognosis. Chest 147: 165-172, 2015
47. Tothill RW, Tinker AV, George J, Brown R, Fox SB, Lade S, Johnson DS, Trivett MK, Etemadmoghadam D, Locandro B, et al: Novel molecular subtypes of serous and endometrioid ovarian cancer linked to clinical outcome. Clin Cancer Res 14: 5198-5208, 2008.

48. Crosas-Molist E, Bertran E and Fabregat I: Cross-Talk Between TGF- $\beta$ and NADPH oxidases during liver fibrosis and hepatocarcinogenesis. Curr Pharm Des 21: 5964-5976, 2015.

49. Dhanasekaran R, Nakamura I, Hu C,Chen G, Oseini AM, Seven ES, Miamen AG, Moser CD, Zhou W, van Kuppevelt TH, et al: Activation of the transforming growth factor- $\beta / S M A D$ transcriptional pathway underlies a novel tumor-promoting role of sulfatase 1 in hepatocellular carcinoma. Hepatology 61: 1269-1283, 2015.

50. Liu J, Chen S, Wang W, Ning BF, Chen F, Shen W, Ding J, Chen W, Xie WF and Zhang X: Cancer-associated fibroblasts promote hepatocellular carcinoma metastasis through chemokine-activated hedgehog and TGF- $\beta$ pathways. Cancer Lett 379: 49-59, 2016.

51. Kroening S, Solomovitch S, Sachs M, Wullich B and Goppelt-Struebe M: Regulation of connective tissue growth factor (CTGF) by hepatocyte growth factor in human tubular epithelial cells. Nephrol Dial Transplant 24: 755-762, 2009.

52. Tsai CC, Wu SB, Kau HC and Wei YH: Essential role of connective tissue growth factor (CTGF) in transforming growth factor- $\beta 1$ (TGF- $\beta 1$ )-induced myofibroblast transdifferentiation from Graves' orbital fibroblasts. Sci Rep 8: 7276, 2018.

This work is licensed under a Creative Commons Attribution-NonCommercial-NoDerivatives 4.0 International (CC BY-NC-ND 4.0) License. 